\title{
Preservation of hepatic branch of the vagus nerve reduces the risk of gallstone formation after gastrectomy
}

\author{
Chao-Jie Wang ${ }^{1,2}$. Seong-Ho Kong ${ }^{1} \oplus$. Ji-Hyeon Park ${ }^{1}$. Jong-Ho Choi ${ }^{1}$. Shin-Hoo Park ${ }^{1}$. Chun-Chao Zhu ${ }^{2}$. \\ Fadhel Alzahrani ${ }^{1,5} \cdot$ Khalid Alzahrani $^{1,6} \cdot$ Yun-Suhk Suh ${ }^{1,3} \cdot$ Do-Joong Park ${ }^{1} \cdot$ Hyuk-Joon Lee $^{1,4} \cdot$ Hui Cao $^{2}$. \\ Han-Kwang Yang ${ }^{1,4}$
}

Received: 20 May 2020 / Accepted: 11 July 2020 / Published online: 23 July 2020

(c) The International Gastric Cancer Association and The Japanese Gastric Cancer Association 2020

\begin{abstract}
Background Injury to the vagus nerve has been proposed to be associated with occurrence of gallstones after gastrectomy. We investigated the effect of preservation of hepatic branch of the vagus nerve on prevention of gallstones during laparoscopic distal (LDG) and pylorus-preserving gastrectomy (LPPG).

Methods Preservation of the vagus nerve was reviewed of cT1N0M0 gastric cancer patients underwent LDG $(n=323)$ and LPPG $(n=144)$ during 2016-2017. Presence of gallstones was evaluated by ultrasonography (US) and computed tomography $(\mathrm{CT})$. Incidences of gallstones were compared between the nerve preserved (h-DG, h-PPG) group and sacrificed (s-DG, s-PPG) group. Clinicopathological features were also compared.

Results The 3-year cumulative incidence of gallstones was lower in the h-DG $(2.7 \%, n=85)$ than the s-DG $(14.6 \%, n=238)$ $(p=0.017)$ and lower in the h-PPG $(1.6 \%, n=123)$ than the s-PPG $(12.9 \%, n=21)(p=0.004)$. Overall postoperative complication rate was similar between the h-DG and s-DG $(p=0.861)$ as well as between the h-PPG and s-PPG $(p=0.768)$. The number of retrieved lymph nodes station \#1 and 3-year recurrence-free survival were not significantly different between the preserved group and sacrificed group. Injury to the vagus nerve $(p=0.001)$ and high body mass index (BMI) $\left(\geq 27.5 \mathrm{~kg} / \mathrm{m}^{2}\right)$ $(p=0.040)$ were found to be independent risk factors of gallstone formation in multivariate analysis.

Conclusions Preservation of hepatic branch of the vagus nerve can be recommended for LDG as well as LPPG of early gastric cancer patients to reduce postoperative gallstone formation.
\end{abstract}

Keywords Hepatic branch of the vagus nerve $\cdot$ Gallstones · Function-preserving gastrectomy $\cdot$ Early gastric cancer

Electronic supplementary material The online version of this article (https://doi.org/10.1007/s10120-020-01106-z) contains supplementary material, which is available to authorized users.

Seong-Ho Kong

seongho.kong@snu.ac.kr

Hui Cao

caohuishcn@hotmail.com

1 Department of Surgery, Seoul National University Hospital, 101, Daehak-ro, Jongno-gu, Seoul 03080, Korea

2 Department of Gastrointestinal Surgery, Ren Ji Hospital, School of Medicine, Shanghai Jiao Tong University, No. 160 $\mathrm{Pu}$ Jian Road, Shanghai 200127, China

\section{Introduction}

With the development of endoscopic screening program and comprehensive treatment, the prognosis of gastric cancer has significantly improved in several eastern countries, especially in Korea and Japan [1-3]. Therefore, the surgeons started to pay more attention to improve the functional

3 Department of Surgery, Seoul National University Bundang Hospital, Seongnam, Korea

4 Cancer Research Institute, Seoul National University, Seoul, Korea

5 Department of Surgery, King Faisal Hospital, Makkah, Saudi Arabia

6 Taif University, Taif, Saudi Arabia 
outcome and quality of life (QoL) without compromising oncologic safety [4, 5]. Laparoscopic pylorus-preserving gastrectomy (LPPG) is a good example of function-preserving surgery and has gained popularity in East Asian countries [6,7].

Cholelithiasis is known as one of the long-term complications of gastrectomy [8-13]. The incidence of gallbladder stones (GBS) after gastrectomy, as reported in many studies, ranges from 5.2 to $15.3 \%$ in Korea [9-11], $7.4 \%$ in China [12], and $13.6 \%$ in Japan [13]. Recently, a longitudinal follow-up study in Korea showed that post-gastrectomy patients might exhibit higher risk of symptomatic gallstones compared to matched normal population (adjusted $\mathrm{HR}=1.77$ ) [14]. In addition, the incidence of common bile duct stones (CBDS) has been reported to be higher in postgastrectomy patients with GBS (44\%) than in the non-gastrectomy patients (11\%) [15]. Although GBS remain silent in most cases, it should be assumed that laparoscopic cholecystectomy (LC) in patients with history of gastrectomy is more complex than general population primarily because of postoperative adhesion [12, 15-17]. Moreover, for cholecystocholedocholithiasis (CCL) patients, the endoscopic retrograde cholangiopancreatography (ERCP) is usually hard to perform because of previous gastrectomy [18].

The pathophysiological mechanism of increase in GBS formation rate after gastrectomy is not yet completely understood. The potential causes of GBS formation might include injury of hepatic branch of the vagus nerve, nonphysiological reconstruction, and distortion of the biliary tract caused by postoperative adhesion [8]. In radical gastrectomy, hepatic branch of the vagus nerve is frequently sacrificed, which is known to be associated with post-gastrectomy cholelithiasis [19]. According to Korean practice guideline for gastric cancer 2018, LPPG can be used for middle-third cT1N0 gastric cancer patients [20]. Preservation of hepatic branch of the vagus nerve is one of the most important procedures of LPPG [6]. Nowadays, the surgical and oncologic safety of LPPG in early gastric cancer (EGC) patients has been well proofed in many retrospective studies $[6,21-23]$. Previous studies have also shown that LPPG can reduce the risk of postoperative GBS formation to the level similar to that of general population, and the level of risk reduction achieved by LPPG is better than that achieved by either laparoscopic distal gastrectomy (LDG) or total gastrectomy (LTG) [22-24].

In this study, we aimed to evaluate whether preservation of hepatic branch of the vagus nerve could reduce the incidence of GBS in patients after gastrectomy and its surgical and oncologic safety in both LDG and LPPG.

\section{Methods}

\section{Patients}

In this retrospective study, we reviewed medical records of patients who underwent LDG or LPPG at Seoul National University Hospital (SNUH) between January 2016 and December 2017. The inclusion criteria for this study were: (1) patients with biopsy proven, at cT1N0M0 staging, according to the 8th edition of American Joint Committee on Cancer (AJCC) TNM classification, (2) patients with no history of cholecystitis, cholelithiasis, or cholecystectomy, (3) patients with no combined resection of gallbladder during gastrectomy, (4) patients with complete surgical video records to prove whether we have preserved hepatic branch of the vagus nerve or not, and (5) patients with follow-up data for more than one year.

\section{Surgical procedures}

Preservation of hepatic branch of the vagus nerve is already an important part of LPPG. While dissecting the lesser omentum, the surgeon divides the lesser omentum below the hepatic branch of anterior vagus nerve so that we can avoid injuring it. Then, during dissection of the \#1 station $\mathrm{LN}$ area, the hepatic branch of anterior vagus nerve also should be carefully protected. Since the branching point is usually proximal to the esophago-gastric junction (EGJ), the \#1 station LN can be sufficiently removed at EGJ level and below without sacrificing the hepatic branch (Fig. 1). Hepatic branch of the vagus nerve usually can be detected during laparoscopy in most cases, but sometimes multiple branches of this nerve might exist. Since 2003, our institution has started performing LPPG for middlethird EGC patients. Before the introduction of preservation of hepatic branch of the vagus nerve to LDG in July 2015, the standardized surgical techniques of nerve protection were routinely used by all surgeons in our institution.

Surgical videos of all cases were reviewed according to following criteria: (1) checking the entire procedures of nerve preservation, starting from the lesser omentum dissection and finishing with \#1 station LN dissection, (2) whether the main thick fibre trunks were cut at potential hepatic branch area, (3) if the hepatic branch had multiple tiny branches, at least $2 \mathrm{~cm}(2 / 3$ head of grasping forceps) of the tissue below the liver should be preserved, (4) \#12 station LN dissection was omitted, (5) there was no Ham-lock clipping or obvious energy device damage to the potential nerve area, (6) whether the hepatic branch was visible (invisible hepatic branch cases were excluded), and (7) the review of surgical videos was performed in 


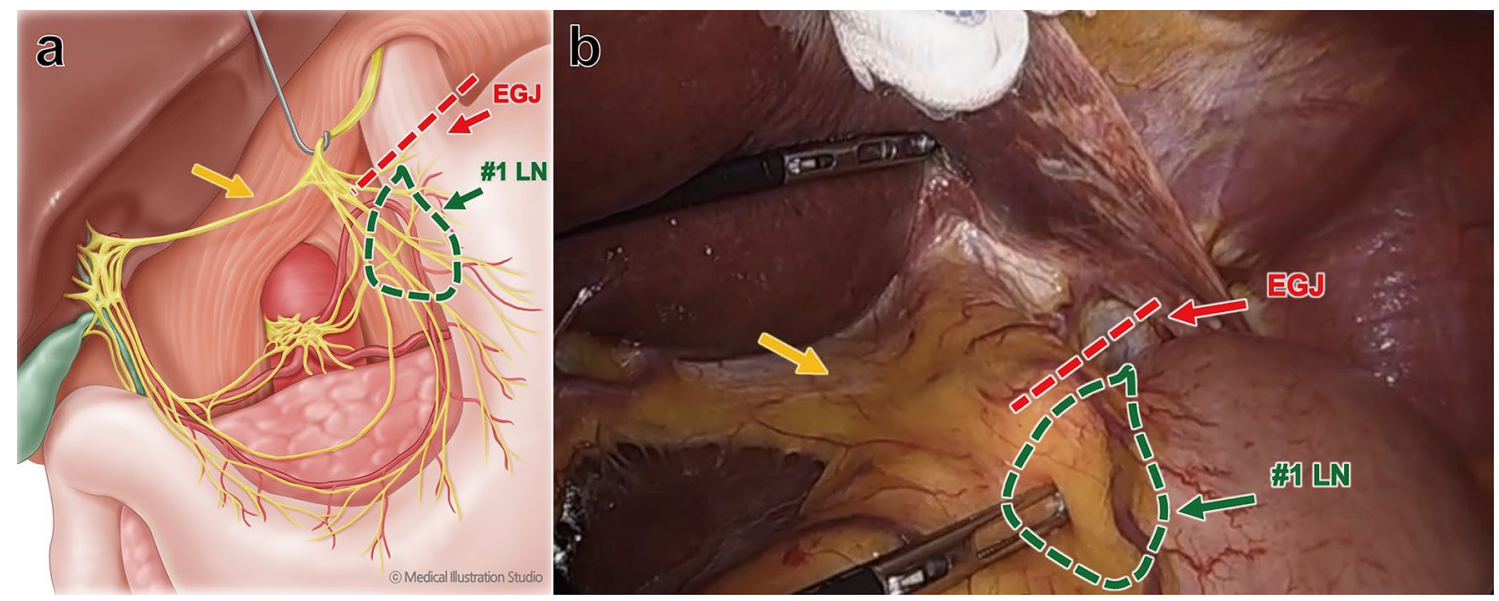

Fig. 1 Preservation of hepatic branch of the vagus nerve. The lesser omentum was dissected below the hepatic branch of anterior vagus nerve (yellow); \#1 LN (green) was sufficiently removed at EGJ level (red) and below

a double-blinded manner by two surgeons (controversial cases were excluded).

\section{Surgical and oncologic outcomes}

The following clinicopathological data were collected and compared: sex, age, body mass index (BMI), body weight, operator, surgical approach, tumor size, pathological TNM stage, and number of resected lymph nodes. Surgical outcomes included duration of surgery, blood loss, postoperative hospital stay, and complications. All complications had been prospectively assessed and reported in the weekly tumor board at SNUH. The severity of complications was classified according to the Clavien-Dindo classification system [25]. Recurrence-free survival was determined by reviewing the results of the follow-up program, including CT and/or US every 3 or 6 months [20].

\section{Diagnosis of cholelithiasis}

During follow-up, CT and/or US should be conducted on post-gastrectomy patients every 6 months. Postoperative GBS formation was diagnosed if the findings of CT and/or US revealed positive observation $\geq 2$ times during the follow-up. Gallbladder sludge, a type of gallbladder abnormality, was also included in this study. For CT(-) GBS detected postoperatively by US and/or MRI, we required at least one US or MRI record to prove the gallbladder was normal before that point. Otherwise, the case should be excluded from the study.

\section{Statistical analysis}

Statistical analyses were performed using SPSS 22.0 (SPSS, Inc., Chicago, IL, USA) and Prism 8 (GraphPad, San Diego,
CA, USA) software. Clinicopathological features and complications were compared using $x^{2}$ test. Quantitative surgical data were compared using Student's $t$ test. Binary logistic regression analysis was used to calculate risk factors of postoperative morbidity. Recurrence-free survival and cumulative incidence of GBS were calculated using the Kaplan-Meier method, and the log-rank test was used to compare the survival distributions. Independent risk factors for postoperative GBS formation were evaluated using logistic regression analysis. A $p$ value less than 0.05 was considered statistically significant.

\section{Results}

\section{Patient characteristics}

During the period of this study, 775 patients received LDG or LPPG in this centre. After applying the eligibility criteria, we excluded 308 cases in the final study cohort (Fig. 2). Among 46 unclear nerve status cases, out of which, 11 were invisible hepatic branch cases and 35 were controversial cases. The concordance rate of two video reviewers was 93.2\%. Among the 467 eligible cases, 323 patients underwent LDG and 144 patients underwent LPPG. Then, surgical video review was used to identify hepatic branch of the vagus nerve preserved subgroup in both the LDG (h-DG, $n=85$ ) and LPPG (h-PPG, $n=123$ ) groups, and the sacrificed subgroup in LDG (s-DG, $n=238$ ) and LPPG (s-PPG, $n=21$ ) groups (Fig. 2). Detailed clinicopathological features and surgical data of these 467 cases are shown in Table 1. $x^{2}$ test revealed a significantly higher proportion of male patients in s-PPG than in the h-PPG group $(p=0.004)$. In the LDG group, we observed significant difference in the nerve preservation rate of three faculties involved in this 
Fig. 2 Patients analysis. $L D G$ laparoscopic distal gastrectomy, $L P P G$ laparoscopic pyloruspreserving gastrectomy, $h-D G$ hepatic branch preserving LDG, $s-D G$ hepatic branch sacrificed LDG, $h-P P G$ hepatic branch preserving LPPG, $s-P P G$ hepatic branch sacrificed LPPG

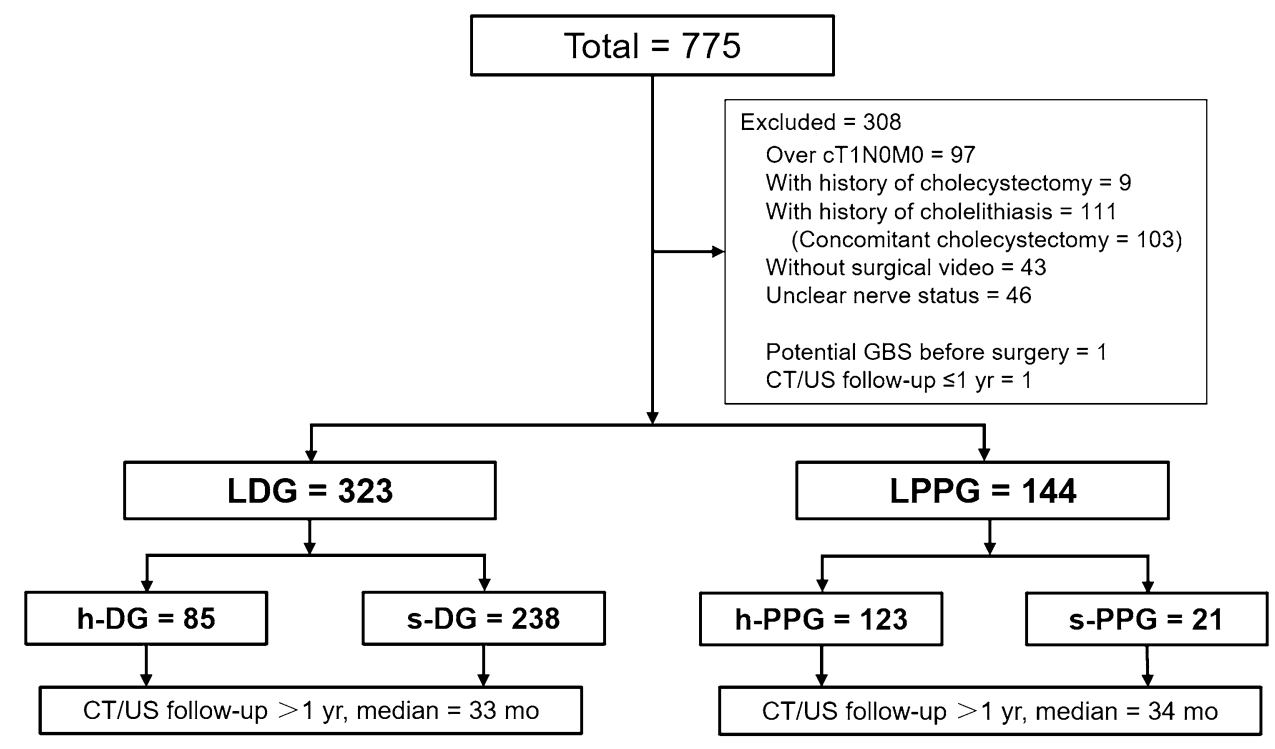

study ( $p<0.001)$. Among h-DG cases, anastomosis of $83.5 \%$ patients was done by Billroth I, which was much higher than the number of patients who underwent Billroth II (14.1\%) and Roux-en-Y $(2.4 \%)(p<0.001)$. There was no significant difference in age, BMI, anastomosis method (mini-laparotomy vs. total-laparoscopic), tumor size, histological type, and pathological TNM stage.

\section{Surgical outcomes}

In both LDG and LPPG, regardless of preservation of hepatic branch of the vagus nerve, there was no significant difference in mean duration of surgery, blood loss, and postoperative duration of hospitalization (Table 1). Analysis of surgery-related complications (Table 2) showed that there was no significant difference with respect to the grade (Grade I to IVa) in LPPG subgroup analysis. Overall morbidity was also similar between the h-PPG and s-PPG groups (19.5 vs. $23.8 \%, p=0.768$ ). Among LDG subgroup, the h-DG group with grade II complications exhibited a significantly higher morbidity rate than did the s-DG group (14.1 vs. $6.7 \%, p=0.045)$. However, the overall morbidity rates of the h-DG and s-DG groups were not significantly different (15.3 vs. $14.7 \%, p=0.861)$. Although delayed gastric emptying (DGE) was more frequent in the LPPG than in the LDG group (9.0 vs. $0.3 \%, p<0.001$ ), no significant difference was found between the h-PPG and s-PPG in LPPG subgroup analysis $(8.9$ vs. $9.5 \%, p=1.000)$. No postoperative mortality was observed in any of the groups. Multivariate analysis showed that operation type (LPPG compared to LDG) was an independent risk factor for the postoperative complication of DGE $(p<0.001$, HR 16.549, 95\% CI 4.842-56.560) (Table 3). Preservation of hepatic branch of the vagus nerve was not responsible for either postoperative complications
( $p=0.564$, HR $0.809,95 \%$ CI $0.393-1.663)$ or DGE after gastrectomy ( $p=0.341$, HR 1.722, 95\% CI 0.562-5.277).

\section{Oncologic safety}

There was no significant difference in the total number of resected LN between the h-DG and s-DG group (35.7 vs. $37.4, p=0.380$ ) or between the h-PPG and s-PPG group (35.4 vs. 33.0, $p=0.386$ ) (Table 1). In LDG, the mean number of examined LN at \#1 station was similar between the h-DG and s-DG groups (2.8 vs. $2.8, p=0.908)$. Similar result was observed for the h-PPG and s-PPG groups $(2.8$ vs. 2.1, $p=0.193$ ). The mean number of resected LN at \#3 station also showed no significant difference between the h-DG and s-DG (4.0 vs. 4.8, $p=0.197)$. In LPPG, similar result was observed for the h-PPG and s-PPG groups (5.4 vs. 5.3, $p=0.941$ ). Since the \#12 station LN dissection was omitted in the h-DG group, the mean number of examined \#12 station LN was 0.0 in the h-DG, which was lower than that in the s-DG (0.4) ( $p=0.003)$ (Fig. 3).

The pathological results of metastatic $\mathrm{LN}$ at each station are shown in Supplementary Table 1. The metastasis rate in \#1 station was lower for the LDG group than that for the LPPG group; however, the difference was not significant ( 0.3 vs. $1.4 \%, p=0.227$ ). For $\# 3$ station, the rate was $4.1 \%$ in LDG and $3.5 \%$ in the LPPG group $(p=0.441)$. The metastatic rate in \#12 station, where dissection is omitted in the h-DG was also $0.0 \%$ in the s-DG.

During follow-up, recurrence was observed in six patients: five patients in the s-DG group and one patient in the h-PPG group. One case in the s-DG group was diagnosed as pT2N0, the rest were all at pT1 stage without LN metastasis. Neither anastomotic site nor regional LN recurrences have been found in either group (Supplementary 
Table 1 Patient's clinicopathologic features and surgical data

\begin{tabular}{|c|c|c|c|c|c|c|}
\hline \multirow[t]{2}{*}{ Characteristics } & \multicolumn{2}{|l|}{ LDG $(n=323)$} & \multicolumn{2}{|l|}{ LPPG $(n=144)$} & \multicolumn{2}{|l|}{$p$ value } \\
\hline & h-DG $(n=85)$ & $\mathrm{s}-\mathrm{DG}(n=238)$ & h-PPG $(n=123)$ & s-PPG $(n=21)$ & $\mathrm{LDG}^{\mathrm{a}}$ & $\mathrm{LPPG}^{\mathrm{b}}$ \\
\hline \multicolumn{7}{|l|}{ Sex } \\
\hline Male & $54(63.5 \%)$ & $149(62.6 \%)$ & $51(41.5 \%)$ & $16(76.2 \%)$ & 0.897 & 0.004 \\
\hline Female & $31(36.5 \%)$ & $89(37.4 \%)$ & $72(58.5 \%)$ & $5(23.8 \%)$ & & \\
\hline \multicolumn{7}{|l|}{ Age } \\
\hline$\leq 60$ & $31(36.5 \%)$ & $117(49.2 \%)$ & $64(52.0 \%)$ & $14(66.7 \%)$ & 0.057 & 0.244 \\
\hline$>60$ & $54(63.5 \%)$ & $121(50.8 \%)$ & $59(48.0 \%)$ & $7(33.3 \%)$ & & \\
\hline \multicolumn{7}{|l|}{ BMI } \\
\hline$<25$ & $47(55.3 \%)$ & $156(65.5 \%)$ & $83(67.5 \%)$ & $13(61.9 \%)$ & 0.116 & 0.624 \\
\hline$\geq 25$ & $38(44.7 \%)$ & $82(34.5 \%)$ & $40(32.5 \%)$ & $8(38.1 \%)$ & & \\
\hline \multicolumn{7}{|l|}{ Operator } \\
\hline A & $39(45.9 \%)$ & $59(24.8 \%)$ & $78(63.4 \%)$ & $18(85.7 \%)$ & $<0.001$ & 0.086 \\
\hline B & $9(10.6 \%)$ & $164(68.9 \%)$ & $27(22.0 \%)$ & $3(14.3 \%)$ & & \\
\hline $\mathrm{C}$ & $37(43.5 \%)$ & $15(6.3 \%)$ & $18(14.6 \%)$ & $0(0.0 \%)$ & & \\
\hline \multicolumn{7}{|l|}{ Anastomosis type } \\
\hline Billroth I & $71(83.5 \%)$ & $129(54.2 \%)$ & & & $<0.001$ & N/A \\
\hline Brillroth II & $12(14.1 \%)$ & $104(43.7 \%)$ & & & & \\
\hline Roux-en-Y & $2(2.4 \%)$ & $5(2.1 \%)$ & & & & \\
\hline Gastrogastrostomy & & & $123(100.0 \%)$ & $21(100.0 \%)$ & & \\
\hline \multicolumn{7}{|l|}{ Anastomosis method } \\
\hline Mini-laparotomy & $35(41.2 \%)$ & $89(37.4 \%)$ & $106(86.2 \%)$ & $18(85.7 \%)$ & 0.604 & 1.000 \\
\hline Total-laparoscopic & $50(58.8 \%)$ & $149(62.6 \%)$ & $17(13.8 \%)$ & $3(14.3 \%)$ & & \\
\hline \multicolumn{7}{|l|}{ Tumor size } \\
\hline$<2 \mathrm{~cm}$ & $26(30.6 \%)$ & $81(34.0 \%)$ & $56(45.5 \%)$ & $12(57.1 \%)$ & 0.785 & 0.516 \\
\hline $2-5 \mathrm{~cm}$ & $54(63.5 \%)$ & $141(59.2 \%)$ & $64(52.0 \%)$ & $9(42.9 \%)$ & & \\
\hline$>5 \mathrm{~cm}$ & $5(5.9 \%)$ & $16(6.7 \%)$ & $3(2.4 \%)$ & $0(0.0 \%)$ & & \\
\hline \multicolumn{7}{|l|}{ Histological type } \\
\hline Differentiated & $36(42.4 \%)$ & $96(40.3 \%)$ & $52(42.3 \%)$ & $10(47.6 \%)$ & 0.797 & 0.643 \\
\hline Undifferentiated & $49(57.6 \%)$ & $142(59.7 \%)$ & $71(57.7 \%)$ & $11(52.4 \%)$ & & \\
\hline \multicolumn{7}{|l|}{ T stage } \\
\hline pT1a & $46(54.1 \%)$ & $128(53.8 \%)$ & $80(65.1 \%)$ & $16(76.2 \%)$ & 0.685 & 0.475 \\
\hline pT1b & $35(41.2 \%)$ & $90(37.8 \%)$ & $38(30.9 \%)$ & $5(23.8 \%)$ & & \\
\hline pT2 & $4(4.7 \%)$ & $19(8.0 \%)$ & $4(3.2 \%)$ & $0(0.0 \%)$ & & \\
\hline pT3 & $0(0.0 \%)$ & $1(0.4 \%)$ & $1(0.8 \%)$ & $0(0.0 \%)$ & & \\
\hline \multicolumn{7}{|l|}{$\mathrm{N}$ stage } \\
\hline pN0 & $75(88.2 \%)$ & $214(89.9 \%)$ & $113(91.9 \%)$ & $20(95.2 \%)$ & 0.964 & 0.667 \\
\hline $\mathrm{pN} 1$ & $7(8.2 \%)$ & $17(7.1 \%)$ & $3(2.4 \%)$ & $0(0.0 \%)$ & & \\
\hline $\mathrm{pN} 2$ & $2(2.4 \%)$ & $4(1.7 \%)$ & $4(3.3 \%)$ & $0(0.0 \%)$ & & \\
\hline $\mathrm{pN} 3$ & $1(1.2 \%)$ & $3(1.3 \%)$ & $3(2.4 \%)$ & $1(4.8 \%)$ & & \\
\hline \multicolumn{7}{|l|}{ TNM (8th AJCC) } \\
\hline p Stage I & $82(96.5 \%)$ & $230(96.6 \%)$ & $116(94.3 \%)$ & $20(95.2 \%)$ & 0.989 & 0.834 \\
\hline p Stage II & $2(2.4 \%)$ & $5(2.1 \%)$ & $5(4.1 \%)$ & $1(4.8 \%)$ & & \\
\hline p Stage III & $1(1.2 \%)$ & $3(1.3 \%)$ & $2(1.6 \%)$ & $0(0.0 \%)$ & & \\
\hline Body weight (kg) & $64.2 \pm 11.8$ & $63.1 \pm 10.1$ & $61.1 \pm 9.7$ & $62.8 \pm 10.3$ & 0.427 & 0.239 \\
\hline Operation time (min) & $224 \pm 61$ & $220 \pm 59$ & $211 \pm 56$ & $210 \pm 48$ & 0.692 & 0.984 \\
\hline Blood loss (ml) & $135 \pm 195$ & $143 \pm 181$ & $104 \pm 103$ & $90 \pm 94$ & 0.750 & 0.553 \\
\hline Hospitalization stay (days) & $8.7 \pm 5.1$ & $9.6 \pm 7.8$ & $10.2 \pm 7.6$ & $9.2 \pm 4.8$ & 0.275 & 0.571 \\
\hline Resected LN & $35.7 \pm 13.1$ & $37.4 \pm 13.9$ & $35.4 \pm 11.4$ & $33.0 \pm 13.7$ & 0.380 & 0.386 \\
\hline
\end{tabular}

Statistical significant values are shown in bold

N/A not assessable because of limited number of cases

${ }^{a}$ Comparison of h-DG and s-DG in LDG

${ }^{\mathrm{b}}$ Comparison of h-PPG and s-PPG in LPPG 
Table 2 Postoperative morbidity

\begin{tabular}{|c|c|c|c|c|c|c|}
\hline \multirow[t]{2}{*}{ Clavien-Dindo Classification } & \multicolumn{2}{|l|}{ LDG $(n=323)$} & \multicolumn{2}{|l|}{ LPPG $(n=144)$} & \multicolumn{2}{|c|}{$p$ value* } \\
\hline & h-DG $(n=85)$ & s-DG $(n=238)$ & h-PPG $(n=123)$ & s-PPG $(n=21)$ & $\mathrm{LDG}^{\mathrm{a}}$ & $\mathrm{LPPG}^{\mathrm{b}}$ \\
\hline \multicolumn{7}{|l|}{ Events with complication } \\
\hline Grade I & $0(0.0 \%)$ & $5(2.1 \%)$ & $0(0.0 \%)$ & $0(0.0 \%)$ & 0.331 & N/A \\
\hline Wound & $0(0.0 \%)$ & $2(0.8 \%)$ & $0(0.0 \%)$ & $0(0.0 \%)$ & & \\
\hline Anastomosis stenosis & $0(0.0 \%)$ & $1(0.4 \%)$ & $0(0.0 \%)$ & $0(0.0 \%)$ & & \\
\hline Ileus/motility disorder & $0(0.0 \%)$ & $1(0.4 \%)$ & $0(0.0 \%)$ & $0(0.0 \%)$ & & \\
\hline Urinary & $0(0.0 \%)$ & $1(0.4 \%)$ & $0(0.0 \%)$ & $0(0.0 \%)$ & & \\
\hline Grade II & $12(14.1 \%)$ & $16(6.7 \%)$ & $15(12.2 \%)$ & $3(14.3 \%)$ & 0.045 & 0.728 \\
\hline Wound & $0(0.0 \%)$ & $1(0.4 \%)$ & $0(0.0 \%)$ & $0(0.0 \%)$ & & \\
\hline Fluid collection & $4(4.7 \%)$ & $2(0.8 \%)$ & $2(1.6 \%)$ & $2(9.5 \%)$ & & \\
\hline Intra-abdominal bleeding & $0(0.0 \%)$ & $1(0.4 \%)$ & $1(0.8 \%)$ & $0(0.0 \%)$ & & \\
\hline Luminal bleeding & $0(0.0 \%)$ & $0(0.0 \%)$ & $2(1.6 \%)$ & $0(0.0 \%)$ & & \\
\hline Anastomosis stenosis & $0(0.0 \%)$ & $1(0.4 \%)$ & $0(0.0 \%)$ & $0(0.0 \%)$ & & \\
\hline Delayed gastric emptying & $0(0.0 \%)$ & $1(0.4 \%)$ & $0(0.0 \%)$ & $0(0.0 \%)$ & & \\
\hline Ileus/motility disorder & $3(3.5 \%)$ & $1(0.4 \%)$ & $1(0.8 \%)$ & $0(0.0 \%)$ & & \\
\hline Pulmonary & $1(1.2 \%)$ & $3(1.3 \%)$ & $3(2.4 \%)$ & $1(4.8 \%)$ & & \\
\hline Urinary & $1(1.2 \%)$ & $0(0.0 \%)$ & $1(0.8 \%)$ & $0(0.0 \%)$ & & \\
\hline Renal & $0(0.0 \%)$ & $1(0.4 \%)$ & $0(0.0 \%)$ & $0(0.0 \%)$ & & \\
\hline Hepatobiliary & $0(0.0 \%)$ & $0(0.0 \%)$ & $1(0.8 \%)$ & $0(0.0 \%)$ & & \\
\hline GI & $1(1.2 \%)$ & $1(0.4 \%)$ & $1(0.8 \%)$ & $0(0.0 \%)$ & & \\
\hline Other infection & $0(0.0 \%)$ & $1(0.4 \%)$ & $3(2.4 \%)$ & $0(0.0 \%)$ & & \\
\hline Cardiac & $0(0.0 \%)$ & $1(0.4 \%)$ & $0(0.0 \%)$ & $0(0.0 \%)$ & & \\
\hline Vascular & $2(2.3 \%)$ & $2(0.8 \%)$ & $0(0.0 \%)$ & $0(0.0 \%)$ & & \\
\hline Grade IIIa & $6(7.1 \%)$ & $18(7.6 \%)$ & $17(13.8 \%)$ & $2(9.5 \%)$ & 1.000 & 0.740 \\
\hline Wound & $0(0.0 \%)$ & $2(0.8 \%)$ & $2(1.6 \%)$ & $0(0.0 \%)$ & & \\
\hline Fluid collection & $0(0.0 \%)$ & $3(1.3 \%)$ & $2(1.6 \%)$ & $0(0.0 \%)$ & & \\
\hline Intra-abdominal bleeding & $0(0.0 \%)$ & $1(0.4 \%)$ & $0(0.0 \%)$ & $0(0.0 \%)$ & & \\
\hline Luminal bleeding & $0(0.0 \%)$ & $1(0.4 \%)$ & $0(0.0 \%)$ & $0(0.0 \%)$ & & \\
\hline Anastomosis stenosis & $2(2.3 \%)$ & $6(2.5 \%)$ & $0(0.0 \%)$ & $0(0.0 \%)$ & & \\
\hline Delayed gastric emptying & $0(0.0 \%)$ & $0(0.0 \%)$ & $11(8.9 \%)$ & $2(9.5 \%)$ & & \\
\hline Anastomosis leakage & $2(2.3 \%)$ & $3(1.3 \%)$ & $2(1.6 \%)$ & $0(0.0 \%)$ & & \\
\hline Duodenal stump leakage & $0(0.0 \%)$ & $2(0.8 \%)$ & $0(0.0 \%)$ & $0(0.0 \%)$ & & \\
\hline Pulmonary & $1(1.2 \%)$ & $0(0.0 \%)$ & $0(0.0 \%)$ & $0(0.0 \%)$ & & \\
\hline Renal & $1(1.2 \%)$ & $0(0.0 \%)$ & $0(0.0 \%)$ & $0(0.0 \%)$ & & \\
\hline Grade IIIb & $0(0.0 \%)$ & $1(0.4 \%)$ & $0(0.0 \%)$ & $0(0.0 \%)$ & 1.000 & N/A \\
\hline Wound & $0(0.0 \%)$ & $1(0.4 \%)$ & $0(0.0 \%)$ & $0(0.0 \%)$ & & \\
\hline Grade IVa & $0(0.0 \%)$ & $5(2.1 \%)$ & $0(0.0 \%)$ & $0(0.0 \%)$ & 0.331 & N/A \\
\hline Intra-abdominal bleeding & $0(0.0 \%)$ & $3(1.3 \%)$ & $0(0.0 \%)$ & $0(0.0 \%)$ & & \\
\hline Luminal bleeding & $0(0.0 \%)$ & $1(0.4 \%)$ & $0(0.0 \%)$ & $0(0.0 \%)$ & & \\
\hline Cardiac & $0(0.0 \%)$ & $1(0.4 \%)$ & $0(0.0 \%)$ & $0(0.0 \%)$ & & \\
\hline \multicolumn{7}{|l|}{ No. patients with complication } \\
\hline All complications & $13 / 85(15.3 \%)$ & $35 / 238(14.7 \%)$ & $24 / 123(19.5 \%)$ & $5 / 21(23.8 \%)$ & 0.861 & 0.768 \\
\hline III, IV complications & $6 / 85(7.1 \%)$ & $20 / 238(8.4 \%)$ & $15 / 123(12.1 \%)$ & $2 / 21(9.5 \%)$ & 0.819 & 1.000 \\
\hline Delayed gastric emptying & $0 / 85(0.0 \%)$ & $1 / 238(0.4 \%)$ & $11 / 123(8.9 \%)$ & $2 / 21(9.5 \%)$ & 1.000 & 1.000 \\
\hline Complications except for DGE & $13 / 85(15.3 \%)$ & $34 / 238(14.3 \%)$ & $13 / 123(10.5 \%)$ & $3 / 21(14.3 \%)$ & 0.858 & 0.705 \\
\hline
\end{tabular}

N/A not assessable because of limited number of cases

* $p$ value was calculated using the number of patients with each grade of complication. Statistical significant values is shown in bold

${ }^{\mathrm{a}}$ Comparison of h-DG and s-DG in LDG

${ }^{b}$ Comparison of h-PPG and s-PPG in LPPG 
Table 3 Risk factors of postoperative morbidity by multivariate analysis
Fig. 3 The mean number of examined lymph nodes in each lymph node station. a LDG group, b LPPG group. * $p<0.05$

\begin{tabular}{|c|c|c|c|c|c|}
\hline \multirow[t]{2}{*}{ Characteristics } & \multirow[t]{2}{*}{$N$} & \multicolumn{2}{|l|}{ Complication } & \multicolumn{2}{|l|}{ Delayed gastric Emptying } \\
\hline & & HR $(95 \% \mathrm{CI})$ & $p$ value & HR $(95 \%$ CI $)$ & $p$ value \\
\hline \multicolumn{6}{|l|}{ Sex } \\
\hline Male & 270 & 1.000 & & 1.000 & \\
\hline Female & 197 & $0.689(0.395-1.203)$ & 0.190 & $1.576(0.692-3.589)$ & 0.279 \\
\hline \multicolumn{6}{|l|}{ Age } \\
\hline$\leq 60$ & 226 & 1.000 & & 1.000 & \\
\hline$>60$ & 241 & $1.072(0.625-1.840)$ & 0.800 & $1.692(0.752-3.808)$ & 0.204 \\
\hline \multicolumn{6}{|l|}{ BMI } \\
\hline$<25 \mathrm{~kg} / \mathrm{m}^{2}$ & 299 & 1.000 & & 1.000 & \\
\hline$\geq 25 \mathrm{~kg} / \mathrm{m}^{2}$ & 168 & $1.530(0.893-2.623)$ & 0.122 & $2.056(0.923-4.580)$ & 0.078 \\
\hline \multicolumn{6}{|l|}{ Operator } \\
\hline A & 194 & 1.000 & & 1.000 & \\
\hline B & 203 & $1.202(0.631-2.291)$ & 0.576 & $2.069(0.814-5.259)$ & 0.127 \\
\hline $\mathrm{C}$ & 70 & $1.136(0.483-2.668)$ & 0.771 & $2.665(0.761-9.335)$ & 0.126 \\
\hline Operation time (min) & 467 & $0.984(0.846-1.145)$ & 0.838 & $0.892(0.735-1.082)$ & 0.245 \\
\hline Blood loss (ml) & 467 & $0.993(0.977-1.008)$ & 0.351 & $1.005(0.984-1.027)$ & 0.642 \\
\hline \multicolumn{6}{|l|}{ Operation type } \\
\hline LDG & 323 & 1.000 & & 1.000 & \\
\hline LPPG & 144 & $1.702(0.857-3.381)$ & 0.129 & $16.549(4.842-56.560)$ & $<0.001$ \\
\hline \multicolumn{6}{|l|}{ Hepatic vagus nerve } \\
\hline Preserved & 208 & 1.000 & & 1.000 & \\
\hline Sacrificed & 259 & $0.809(0.393-1.663)$ & 0.564 & $1.722(0.562-5.277)$ & 0.341 \\
\hline
\end{tabular}

Statistical significant values is shown in bold

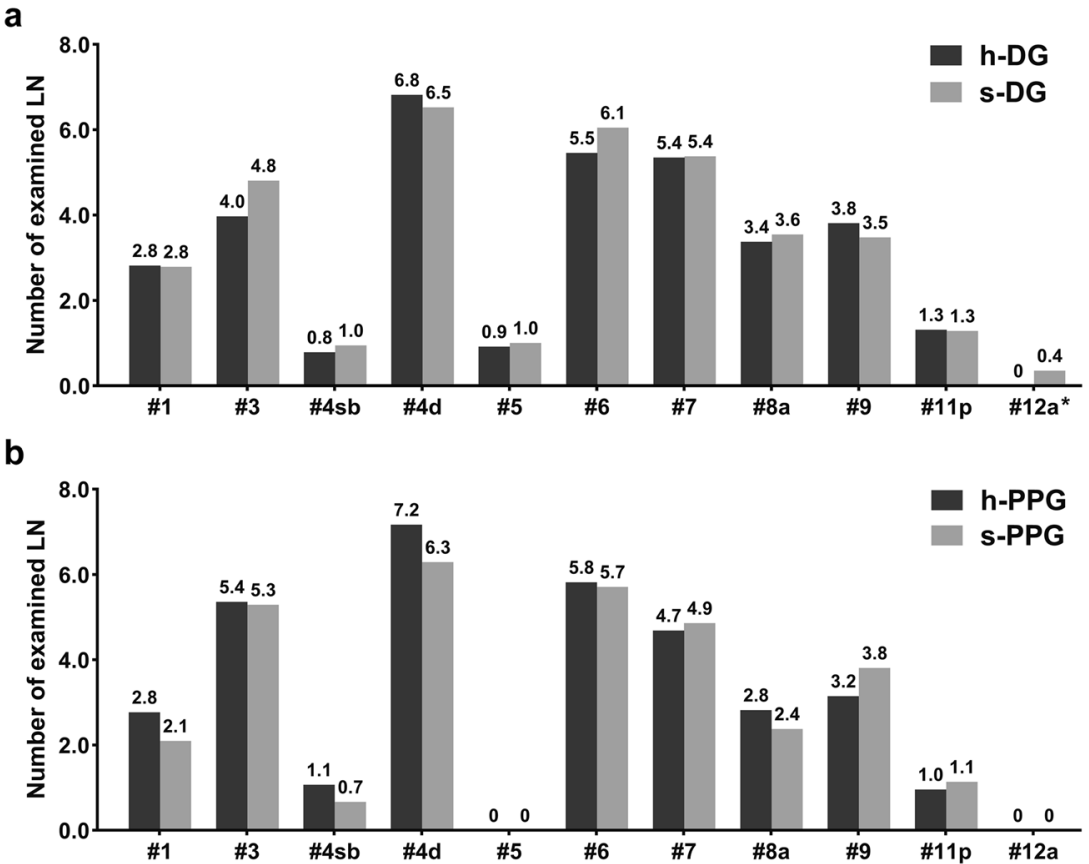

Table 2). The 3-year recurrence-free survival rate was $100.0 \%$ in the h-DG and $97.8 \%$ in the s-DG $(p=0.177)$. The median follow-up period was 32.9 months for the h-DG group and 34.3 months for the s-DG group (Fig. $4 a$ ).
In analysis of LPPG, the 3-year recurrence-free survival rate in the h-PPG group was comparable to that of the s-PPG group (99.0 vs. $100.0 \%, p=0.759$ ). Median 

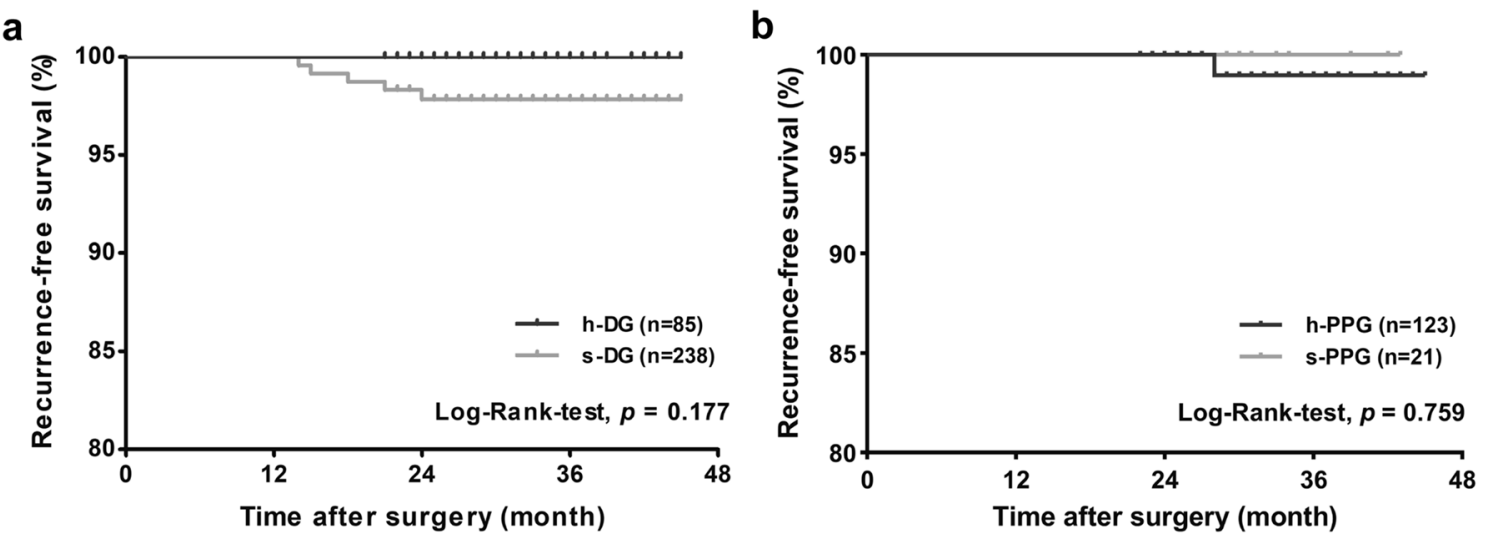

Fig. 4 Recurrence-free survival after gastrectomy. a Comparison of h-DG and s-DG, b comparison of h-PPG and s-PPG

follow-up period was 34.7 months for the h-PPG and 31.2 months for the s-PPG group (Fig. 4b).

\section{Gallbladder stone formation}

Among 468 cases with follow-up data, 35 patients experienced cholelithiasis after gastrectomy. CT(+) GBS was observed in $34.3 \%(12 / 35)$ patients, while US(+) GBS was observed in $93.8 \%(30 / 32)$ patients. With further analysis of the $23 \mathrm{CT}(-) / \mathrm{US}(+)$ GBS cases diagnosed after gastrectomy, four exhibited postoperatively GBS formation because they had US records before surgery to confirm that they were free from cholelithiasis preoperatively. Eighteen of the rest 19 cases also exhibited postoperatively GBS formation because of their negative US records at postoperative 6 months. Finally, we excluded one case from further analysis according to the diagnosis criteria (Fig. 2).

Using 467 cases enrolled for postoperative GBS formation analysis, the overall 3-year cumulative incidence of GBS was $10.2 \%$, and the incidence was significantly higher in the LDG (11.7\%) group than in the LPPG (3.0\%) group $(p=0.010)$. Furthermore, the cumulative curve showed that 3-year cumulative incidence of GB stone/sludge of the s-DG group was much higher than that of the h-DG group ( 14.6 vs. $2.7 \%, p=0.017$ ), and incidence in the s-PPG group was higher than that of the h-PPG group (12.9 vs. $1.6 \%$, $p=0.004$ ). In addition, among the 208 vagus nerve preserving patients, four experienced GBS formation, and 30 patients had cholelithiasis among the 259 vagus nerve sacrificed cases (3-year cumulative incidence in $\mathrm{h}$-Vagus $=2.0 \%$ and in $\mathrm{s}$-Vagus $=14.7 \%, p<0.001 ;$ Fig. $5 \mathrm{a}-\mathrm{c}$ ). After excluding the cases with sludge, the 3 -year cumulative incidence of GBS in the s-DG group was still higher than of the h-DG group (9.1 vs. $1.5 \%, p=0.049$ ). The incidence of GBS was also higher in the s-PPG group than in the h-PPG group, but the difference was not significant (6.2 vs. $0.0 \%, p=0.056$ ). Among the 467 patients, the h-Vagus group showed lower incidence of GBS formation than the s-Vagus group ( 0.6 vs. $9.0 \%, p=0.001$; Fig. $5 \mathrm{~d}-\mathrm{f})$. The univariate analysis showed that patients with high BMI (BMI $\left.\geq 27.5 \mathrm{~kg} / \mathrm{m}^{2}\right)$, history of LDG or vagus nerve sacrificed surgery, and pT2 -3 or pN1-3 stage exhibited higher risk of postoperative GBS formation (Table 4). However, multivariate analysis showed that only high BMI ( $p=0.040$, HR $2.464,95 \%$ CI $1.041-5.831)$ and injury to the vagus nerve $(p=0.001, \mathrm{HR} 8.782,95 \% \mathrm{CI}$ 2.521-30.594) appeared to be independent risk factors of GBS formation after gastrectomy.

During follow-up, two patients from the s-DG group experienced GBS combined with CBDS, out of which, one only received medical treatment during period of this study and the other received open surgery because of concurrent hepatocellular carcinoma. Three patients from the same group suffered from GBS with acute cholecystitis, out of which, two patients recovered through medical treatment and one received laparoscopic cholecystectomy. No mortality was observed due to biliary disorder during this study.

\section{Discussion}

Although postoperative GBS usually remains silent, patients with symptoms still require medical attention. Moreover, performing LC in patients with a history of gastrectomy is more challenging than performing it in the normal population due to postoperative adhesion [15-17]. For elderly patients, secondary abdominal surgery could prove to be fatal because of their basic illnesses [12]. The above-mentioned factors lead to worse QoL and additional financial burden on EGC patients after gastrectomy, and this situation is prolonged due to their long-term survival. In this retrospective study, the overall 3-year cumulative incidence of GBS was $10.2 \%$ in all 467 patients. Among the patients suffered from GBS, 5.9\% (2/34) of them also presented with CBDS, 8.8\% (3/34) received medical treatment, $2.9 \%(1 / 34)$ 
a

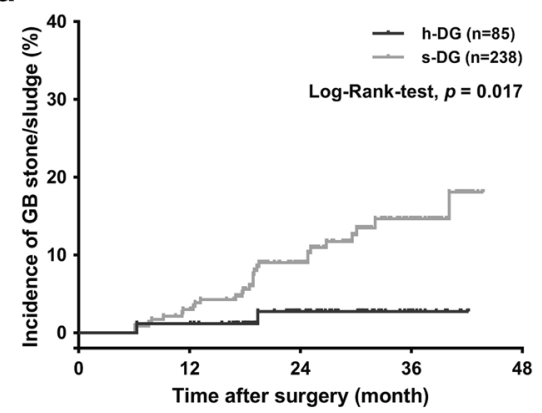

d

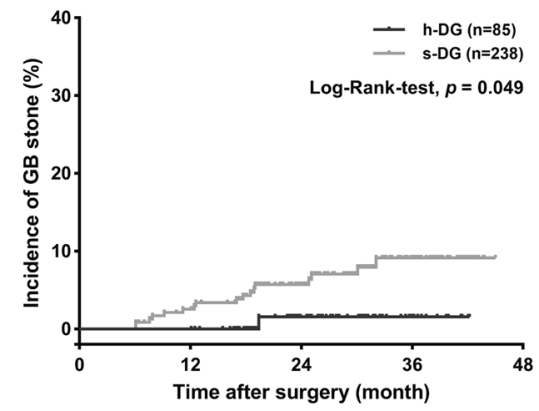

b

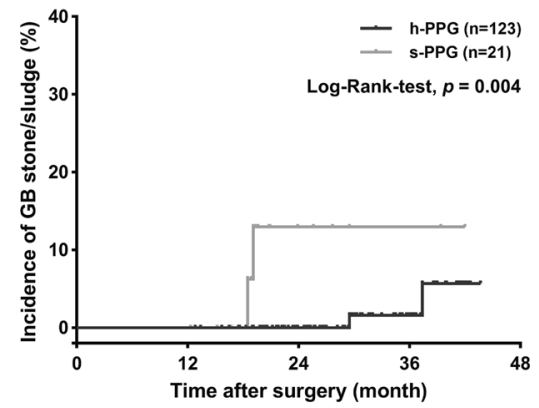

e

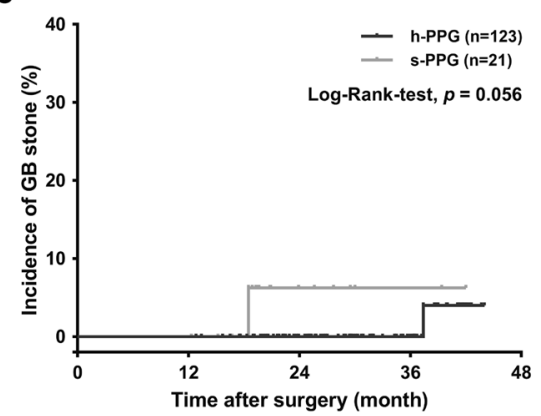

C

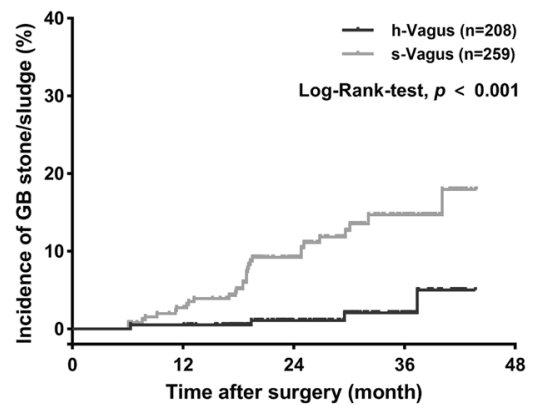

f

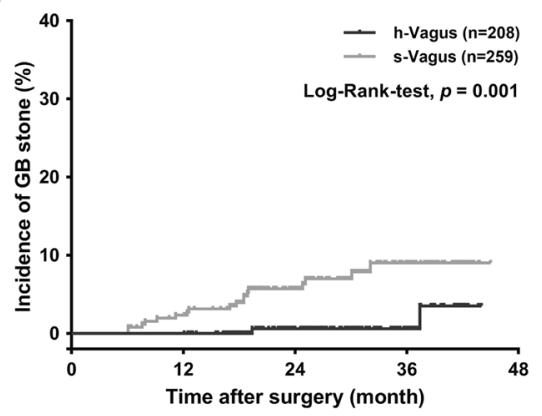

Fig. 5 Cumulative incidence of gallstone after gastrectomy. a-c GBS including sludge, $\mathbf{d}-\mathbf{f}$ GBS excluding sludge

underwent LC, and 2.9\% (1/34) underwent open cholecystectomy. The median follow-up period was 34 months; we believe the incidence could increase in the future.

In LDG, the procedure for preservation of hepatic branch of the vagus nerve has been applied to early gastric cancer patients since mid-2015 in our institute. The preservation procedure was similar to that employed in LPPG, with the only difference of dissection of LN at \#5 station. We selected the patients that underwent the surgery after January 2016 in this study, so we assumed that the standardized surgical techniques and random assignment of patients to each surgeon might minimize the possibility of heterogeneous patient enrolment.

Limitations to lymph node dissection and subsequent oncologic safety have been major concerns of performing vagus nerve-preserving gastrectomy. Preservation of these nerves leads to limitation of $\mathrm{LN}$ dissection at \#1 and \#3 stations and even omission of LN dissection at \#12a station, which might compromise its radical effects of gastrectomy and raise oncologic concerns. As mentioned in Korean practice guideline for gastric cancer 2018, a D1 or $\mathrm{D} 1+$ lymphadenectomy, in which the \#12a LN dissection is not included, is indicated for only cT1N0 tumors [20]. To guarantee the oncologic safety, we strictly forbade the procedure for the preservation of hepatic branch of the vagus nerve, regardless of what nodal involvement was suspected. Our data demonstrated that although \#12a LN dissection was omitted in the vagus nerve preserving group, the probability of LN metastasis to this station was $0.0 \%(0 / 62)$ considering the metastasis rate in the s-DG group. For \#1 and \#3 LN dissection, previous retrospective studies evaluating the result of LPPG in middle-third cT1N0 gastric cancer reported that there was no significant difference in the number of examined LN at \#1 and \#3 between patients undergoing LDG and LPPG [22, 23]. Our data also demonstrated that preservation of hepatic branch of the vagus nerve will affect neither totally resected $\mathrm{LN}$ nor examined $\mathrm{LN}$ at \#1 or \#3 stations between the h-PPG and s-PPG groups. In fact, when performing LPPG for EGC involving the upper third, due to closeness of the tumor, LN \#1 station was always radically resected for oncologic safety [24]. This was the main reason for sacrificing hepatic branch of the vagus nerve in the LPPG group. In cases of LDG, the tumor is usually located in lower third portion. As a result, the metastatic rate at LN \#1 station was even lower in LDG than in LPPG (0.3 vs. 1.4\%). Therefore, we can assume that LDG, with preservation of hepatic branch of the vagus nerve, together with LPPG, is not a compromised procedure for cT1N0M0 EGC patients. The RFS curve also showed that there was no difference in recurrence-free survival rates between the h-DG and s-DG groups or between the h-PPG and s-PPG groups. In addition, the recurrence pattern of five cases in the s-DG group and one case in the h-PPG group showed no regional LN metastasis, which demonstrated the oncologic safety of vagus nerve preserving gastrectomy both for the LDG and LPPG. 
Table 4 Risk factors of postoperative GBS formation in univariate and multivariate analysis

\begin{tabular}{|c|c|c|c|c|c|}
\hline \multirow[t]{2}{*}{ Characteristics } & \multirow[t]{2}{*}{$N$} & \multicolumn{2}{|l|}{ Univariate analysis } & \multicolumn{2}{|l|}{ Multivariate analysis } \\
\hline & & HR $(95 \%$ CI) & $p$ value & HR $(95 \%$ CI $)$ & $p$ value \\
\hline \multicolumn{6}{|l|}{ Sex } \\
\hline Male & 270 & 1.000 & & 1.000 & \\
\hline Female & 197 & $0.822(0.411-1.641)$ & 0.578 & $0.970(0.479-1.964)$ & 0.932 \\
\hline \multicolumn{6}{|l|}{ Age } \\
\hline$\leq 60$ & 226 & 1.000 & & 1.000 & \\
\hline$>60$ & 241 & $1.399(0.706-2.770)$ & 0.336 & $1.360(0.679-2.724)$ & 0.385 \\
\hline \multicolumn{6}{|l|}{ BMI } \\
\hline$<27.5 \mathrm{~kg} / \mathrm{m}^{2}$ & 410 & 1.000 & & 1.000 & \\
\hline$\geq 27.5 \mathrm{~kg} / \mathrm{m}^{2}$ & 57 & $2.455(1.080-5.578)$ & 0.027 & $2.464(1.041-5.831)$ & 0.040 \\
\hline \multicolumn{6}{|l|}{ Operator } \\
\hline A & 194 & 1.000 & & 1.000 & \\
\hline B & 203 & $1.493(0.516-4.318)$ & 0.352 & $1.509(0.491-4.632)$ & 0.472 \\
\hline $\mathrm{C}$ & 70 & $1.429(0.674-3.029)$ & 0.460 & $0.764(0.342-1.707)$ & 0.512 \\
\hline \multicolumn{6}{|l|}{ Operation type } \\
\hline LDG & 323 & 1.000 & & 1.000 & \\
\hline LPPG & 144 & $0.279(0.098-0.792)$ & 0.017 & $1.122(0.316-3.988)$ & 0.859 \\
\hline \multicolumn{6}{|l|}{ T stage } \\
\hline pT1a & 270 & 1.000 & & 1.000 & \\
\hline pT1b & 168 & $2.047(0.582-7.203)$ & 0.264 & $1.359(0.371-4.981)$ & 0.644 \\
\hline pT2-3 & 29 & $2.457(1.201-5.028)$ & 0.014 & $1.949(0.912-4.168)$ & 0.085 \\
\hline \multicolumn{6}{|l|}{$\mathrm{N}$ stage } \\
\hline pNO & 422 & 1.000 & & 1.000 & \\
\hline $\mathrm{pN} 1-3$ & 45 & $2.596(1.130-5.966)$ & 0.025 & $2.251(0.910-5.570)$ & 0.079 \\
\hline \multicolumn{6}{|c|}{ Hepatic vagus nerve } \\
\hline Preserved & 208 & 1.000 & & 1.000 & \\
\hline Sacrificed & 259 & $6.434(2.266-18.267)$ & $<0.001$ & $8.782(2.521-30.594)$ & 0.001 \\
\hline
\end{tabular}

Statistical significant values is shown in bold
We also assessed the surgical safety of nerve preserving procedure. Our data demonstrated that preservation of hepatic branch of the vagus nerve would not make any significant difference in the duration of surgery, blood loss, and duration of hospitalization between the LDG and LPPG groups. In addition, there was no evidence of increase in the postoperative morbidity rates of both the LDG and LPPG groups due to preservation of hepatic branch of the vagus nerve. Previous studies showed that meticulous dissection during preserving procedure might improve retrieval of lymph nodes but could also result in impaired pyloric function [26-28]. In this study, we found that DGE rate in the LPPG group was 9.0\% (13/144) and that in the LDG group was $0.3 \%(1 / 323)$, which was similar to previous study [22]. However, we found no significant difference between the DGE rates of the h-PPG and s-PPG subgroups (8.9 vs. $9.5 \%, \mathrm{p}=1.000$ ). Although the number of patients was limited, we suggested that nerve dysfunction might not be the main cause of postoperative DGE. Multivariate analysis also demonstrated that the operation type (LPPG compared to LDG), but not the status of hepatic branch of vagus nerve (sacrificed compared to preserved), could have increased the risk of DGE after gastrectomy.

With respect to the function-preserving outcomes, previous studies have reported the 3-year cumulative incidence of GBS ranging from 0.0 to $3.2 \%$ after LPPG, which was significantly lower than the incidences obtained after distal and total gastrectomy [22-24]. However, whether the preservation of hepatic branch of the vagus nerve reduced these incidences is still unclear. Some studies also reported that exclusion of duodenum and reconstruction of digestive tract might also responsible for the higher incidence of GBS formation after gastrectomy [12, 13]. In addition, previous studies have reported a higher rate of GBS in patients after Billroth-II and Roux-en-Y reconstruction than that after Billroth-I reconstruction. These studies also demonstrated that change in the pattern of cholecystokinin secretion might decrease the contraction of gallbladder and result in higher risk of GBS $[29,30]$. In our study, we found that the 3 -year cumulative incidence of GBS was significantly higher in the LDG (11.7\%) group than in the LPPG (3.0\%) group $(p=0.010)$, which was consistent with the previous reports. 
With further analysis, we found that the incidence of GBS was also higher in the s-DG (14.6\%) and s-PPG (12.9\%) groups compared with the h-DG (2.7\%) and h-PPG (1.6\%) groups ( $p=0.017$ and $p=0.004$, respectively), which can indirectly support the function of preserved vagus nerve. The multivariate analysis confirmed that the preservation of hepatic branch of the vagus nerve, but not the pylorus preserving procedure, could help reduce the incidence of GBS formation after gastrectomy. Even the patients who underwent LDG can benefit from this nerve preserving procedure and exhibit around nine times less risk of postoperative GBS formation. Moreover, we observed that the 3-year cumulative incidence of GBS was 9.1 and $17.1 \%$ in the LDG group with Billroth-I and Billroth-II reconstruction, respectively; however, the difference was not statistically significant $(p=0.069)$. Although celiac branch of the vagus nerve was sacrificed as a routine procedure during this study, it was reported that no clinical benefits of the preservation of celiac branch of the vagus nerve after LPPG are identified [31]. The incidences obtained in this study were relatively higher than those previously reported because the patients with gallbladder sludge were also included in the present analysis. We believed that the gallbladder sludge was a type of gallbladder abnormality that was related to the previous gastrectomy. The median follow-up period was 34 months. A previous study reported that GBS usually developed within 2 years after surgery, which indicated that the follow-up period in the present study is acceptable for the evaluation of postoperative GBS formation rate [32].

It has been previously shown that female sex, advanced age, obesity, and over-nutrition are all risk factors for gallstone formation [33-36]. However, the association of these risk factors with postoperative GBS formation is still not clear. In our study, we set the cut-off point at BMI $=27.5 \mathrm{~kg} /$ $\mathrm{m}^{2}$. Although BMI $\geq 25.0 \mathrm{~kg} / \mathrm{m}^{2}$ is identified as obesity in Asia-Pacific areas, additional trigger points for public health action was identified $27.5 \mathrm{~kg} / \mathrm{m}^{2}$ as representing high risk [37]. As a result, we found that high BMI also appeared to be an independent predictive factor of GBS formation after gastrectomy. Such obese patients will have 2.5 times higher risk of postoperative GBS formation than the patients with low BMI. The pT2-3 and pN1-3 patients present with a higher risk of postoperative GBS formation, which could be because we tend to perform more exhaustive dissection among these kinds of patients.

In this study, only five patients were diagnosed as symptomatic gallstones, and two of them received cholecystectomy. Although the prophylactic cholecystectomy remains controversial [38, 39], our results showed that most postoperative GBS cases were asymptomatic. For patients with silent GBS before surgery in our institute, whether performing prophylactic cholecystectomy depended on the patient's condition and their own wishes, and most of them had received combined LC because of the relatively higher risk of cholelithiasis after gastrectomy. In the future, we assumed that for those patients without risk factors, such as aging, obesity, and diabetes, preservation of hepatic branch of the vagus nerve can help in preserving their gallbladder too.

This study had several limitations. First, uneven demographic data with respect to sex and surgeons can be a limitation to this retrospective study. Limited number of cases in the s-PPG group might be responsible for uneven demographic data of sex in LPPG analysis. However, sex and surgeons were not independent risk factors for either postoperative morbidity or postoperative GBS formation in this study. Second, the incidence of GBS might have been underestimated because of the predominant use of CT scan for tumor diagnosis. In this study, we set up a criteria for $\mathrm{CT}(-) /$ US(+) GBS diagnosis after surgery to avoid bias. And for potential CT(-) GBS in patients without US reports postoperatively might be a problem, although the number of such cases was limited $(35 / 467,7.5 \%)$. Third limitation is the possibility of unnoticed injuries to the vagus nerve during surgery. Our previous research reported that, although we tried to preserve the nerve, some patients still lost response to hepatic branch of the vagus nerve as revealed by intraoperative neurophysiologic testing [40].

Although we are waiting for the results of a longer followup period of this cohort to confirm the results, we are convinced that the preservation of hepatic branch of the vagus nerve can reduce the incidence of postoperative GBS formation in patients after both LDG and LPPG.

\section{Conclusions}

Preservation of hepatic branch of the vagus nerve can be recommended for LDG as well as LPPG of early gastric cancer patients to reduce postoperative gallstone formation.

Acknowledgements This study is supported by Incubating Program for Clinical Research and Innovation of Ren Ji Hospital, School of Medicine, Shanghai Jiao Tong University (Grant no. PY2018-IIC-06) and National Natural Science Foundation of China International Exchange Project (Grant no. 81811540415). We also deeply appreciate to Medical Art Studio (Sun-Joo Kim and Su-hyun Chea) for medical illustration visualizing our concept.

\section{Compliance with ethical standards}

Conflict of interest The authors declare that they have no conflict of interest.

Ethics statement This study was conducted in accordance with the Ethical Principles for Medical Research Involving Human Subjects, as outlined in the Declaration of Helsinki after the approval of the institutional review board of Seoul National University Hospital. Informed consent was waived by the institutional review board based on its decision that the risk of this study to the patient is minimal. The 
research protocol was approved by the institutional review board of Seoul National University Hospital (No. 2001-150-1097).

\section{References}

1. Bray F, Ferlay J, Soerjomataram I, Siegel RL, Torre LA, Jemal A. Global cancer statistics 2018: GLOBOCAN estimates of incidence and mortality worldwide for 36 cancers in 185 countries. CA Cancer J Clin. 2018;68:394-424.

2. Hamashima C, Systematic Review Group and Guideline Development Group for Gastric Cancer Screening Guidelines. Update version of the Japanese guidelines for gastric cancer screening. Jpn J Clin Oncol. 2018;48:673-83.

3. Jun JK, Choi KS, Lee HY, Suh M, Park B, Song SH, et al. Effectiveness of the Korean National Cancer Screening Program in reducing gastric cancer mortality. Gastroenterology. 2017;152:1319-28.

4. Wang S, Yu S, Xu J, Zhao G. Valuation and prospect of function preserving gastrectomy. Zhonghua Wei Chang Wai Ke Za Zhi. 2017;20:1141-4.

5. Nomura E, Okajima K. Function-preserving gastrectomy for gastric cancer in Japan. World J Gastroenterol. 2016;22:5888-955.

6. Oh SY, Lee HJ, Yang HK. Pylorus-preserving gastrectomy for gastric cancer. J Gastric Cancer. 2016;16:63-71.

7. Shinohara H, Sonoda T, Niki M, Nomura E, Nishiguchi K, Tanigawa N. Laparoscopically-assisted pylorus-preserving gastrectomy with preservation of the vagus nerve. Eur J Surg. 2002;168:55-8.

8. Paik KH, Lee JC, Kim HW, Kang J, Lee YS, Hwang JH, et al. Risk factors for gallstone formation in resected gastric cancer patients. Medicine (Baltimore). 2016. https://doi.org/10.1097/MD.00000 00000003157.

9. Seo GH, Lim CS, Chai YJ. Incidence of gallstones after gastric resection for gastric cancer: a nationwide claims-based study. Ann Surg Treat Res. 2018;95:87-93.

10. Park DJ, Kim KH, Park YS, Ahn SH, Park do J, Kim HH. Risk factors for gallstone formation after surgery for gastric cancer. $\mathrm{J}$ Gastric Cancer. 2016;16:98-104.

11. Jun KH, Kim JH, Kim JJ, Chin HM, Park SM. Retrospective analysis on the gallstone disease after gastrectomy for gastric cancer. Gastroenterol Res Pract. 2015. https://doi. org/10.1155/2015/827864.

12. Liang TJ, Liu SI, Chen YC, Chang PM, Huang WC, Chang HT, et al. Analysis of gallstone disease after gastric cancer surgery. Gastric Cancer. 2017;20:895-903.

13. Kobayashi T, Hisanaga M, Kanehiro H, Yamada Y, Ko S, Nakajima Y. Analysis of risk factors for the development of gallstones after gastrectomy. Br J Surg. 2005;92:1399-403.

14. Kim SY, Bang WJ, Lim H, Lim MS, Kim M, Choi HG. Increased risk of gallstones after gastrectomy: a longitudinal follow-up study using a national sample cohort in korea. Medicine (Baltimore). 2019. https://doi.org/10.1097/MD.0000000000015932.

15. Hashimoto M, Imamura T, Tamura T, Koyama R, Koizumi $Y$, Makuuchi M, et al. Treatment of biliary tract stones after gastrectomy in the era of laparoscopic cholecystectomy. J Hepatobiliary Pancreat Sci. 2016;23:703-7.

16. Wang M, Zhang T, Peng C. Laparoscopic cholecystectomy with previous gastrectomy. J Invest Surg. 2013;26:96-8.

17. Yamamoto H, Hayakawa N, Yamamoto T, Momiyama M, Nagino M. Laparoscopic cholecystectomy in patients with a previous history of gastrectomy. Hepatogastroenterology. 2013;60:443-6.

18. Zhang M, Zhang J, Sun X, Xu J, Zhu J, Yuan W, et al. Clinical analysis of treatment strategies to cholecystocholedocholithiasis patients with previous subtotal or total gastrectomy: a retrospective cohort study. BMC Surg. 2018;18:54.

19. Chen Y, Li Y. Related factors of postoperative gallstone formation after distal gastrectomy: a meta-analysis. Indian J Cancer. 2017;54:43-6.

20. Guideline Committee of the Korean Gastric Cancer Association (KGCA), Development Working Group and Review Panel. Korean practice guideline for gastric cancer: an evidence-based, multidisciplinary approach. J Gastric Cancer. 2018;2019(19):1-48.

21. Tsujiura M, Hiki N, Ohashi M, Nunobe $S$, Kumagai K, Ida S, et al. Should pylorus-preserving gastrectomy be performed for overweight/obese patients with gastric cancer? Gastric Cancer. 2019;22:1247-55.

22. Suh YS, Han DS, Kong SH, Kwon S, Shin CI, Kim WH, et al. Laparoscopy-assisted pylorus-preserving gastrectomy is better than laparoscopy-assisted distal gastrectomy for middle-third early gastric cancer. Ann Surg. 2014;259:485-93.

23. Eom BW, Park B, Yoon HM, Ryu KW, Kim YW. Laparoscopyassisted pylorus-preserving gastrectomy for early gastric cancer: a retrospective study of long-term functional outcomes and quality of life. World J Gastroenterol. 2019;25:5494-504.

24. Zhu CC, Cao H, Berlth F, Xu J, Park SH, Choe HN, et al. Pyloruspreserving gastrectomy for early cancer involving the upper third: can we go higher? Gastric Cancer. 2019;22:881-91.

25. Clavien PA, Barkun J, de Oliveira ML, Vauthey JN, Dindo D, Schulick RD, et al. The Clavien-Dindo classification of surgical complications: five-year experience. Ann Surg. 2009;250:187-96.

26. Nunobe S, Sasako M, Saka M, Fukagawa T, Katai H, Sano T. Symptom evaluation of long-term postoperative outcomes after pylorus-preserving gastrectomy for early gastric cancer. Gastric Cancer. 2007;10:167-72.

27. Jiang X, Hiki N, Nunobe S, Fukunaga T, Kumagai K, Nohara $\mathrm{K}$, et al. Postoperative outcomes and complications after laparoscopy-assisted pylorus-preserving gastrectomy for early gastric cancer. Ann Surg. 2011;253:928-33.

28. Kiyokawa T, Hiki N, Nunobe S, Honda M, Ohashi M, Sano T. Preserving infrapyloric vein reduces postoperative gastric stasis after laparoscopic pylorus-preserving gastrectomy. Langenbecks Arch Surg. 2017;402:49-56.

29. Masclee AA, Jansen JB, Driessen WM, Geuskens LM, Lamers CB. Delayed plasma cholecystokinin and gallbladder responses to intestinal fat in patients with Billroth I and II gastrectomy. Surgery. 1989;106:502-8.

30. Barbara L, Sama C, Morselli Labate AM, Taroni F, Rusticali AG, Festi D, et al. A population study on the prevalence of gallstone disease: the Sirmione study. Hepatology. 1987;7:913-7.

31. Furukawa H, Ohashi M, Honda M, Kumagai K, Nunobe S, Sano $\mathrm{T}$, et al. Preservation of the celiac branch of the vagal nerve for pylorus-preserving gastrectomy: is it meaningful? Gastric Cancer. 2018;21:516-23.

32. Fukagawa T, Katai H, Saka M, Morita S, Sano T, Sasako M. Gallstone formation after gastric cancer surgery. J Gastrointest Surg. 2009;13:886-9.

33. Méndez-Sánchez N, Chavez-Tapia NC, Motola-Kuba D, SanchezLara K, Ponciano-Rodríguez G, Baptista H, et al. Metabolic syndrome as a risk factor for gallstone disease. World J Gastroenterol. 2005;11:1653-7.

34. Tsai CJ, Leitzmann MF, Willett WC, Giovannucci EL. Central adiposity, regional fat distribution, and the risk of cholecystectomy in women. Gut. 2006;55:708-14.

35. Weikert C, Weikert S, Schulze MB, Pischon T, Fritsche A, Bergmann MM, et al. Presence of gallstones or kidney stones and risk of type 2 diabetes. Am J Epidemiol. 2010;171:447-54.

36. Lammert F, Gurusamy K, Ko CW, Miquel JF, Méndez-Sánchez N, Portincasa P, et al. Gallstones. Nat Rev Dis Primers. 2016. https ://doi.org/10.1038/nrdp.2016.24. 
37. WHO Expert Consultation. Appropriate body-mass index for Asian populations and its implications for policy and intervention strategies. Lancet. 2004;363:157-63.

38. Kimura J, Kunisaki C, Takagawa R, Makino H, Ueda M, Ota M, et al. Is routine prophylactic cholecystectomy necessary during gastrectomy for gastric cancer? World J Surg. 2017;41:1047-53.

39. Tan Z, Xie P, Qian H, Yao X. Clinical analysis of prophylactic cholecystectomy during gastrectomy for gastric cancer patients: a retrospective study of 1753 patients. BMC Surg. 2019;19:48.

40. Kong SH, Kim SM, Kim DG, Park KH, Suh YS, Kim TH, et al. Intraoperative neurophysiologic testing of the perigastric vagus nerve branches to evaluate viability and signals along nerve pathways during gastrectomy. J Gastric Cancer. 2019;19:49-61.

Publisher's Note Springer Nature remains neutral with regard to jurisdictional claims in published maps and institutional affiliations. 